\title{
Visual performance and aberration associated with contact lens wear in patients with keratoconus: a pilot study
}

This article was published in the following Dove Press journal:

Clinical Optometry

16 August 2014

Number of times this article has been viewed

Mustafa Abdu

Norhani Mohidin

Bariah Mohd-Ali

Optometry and Vision Science Program, School of Healthcare Sciences, Faculty of Health Science, Universiti Kebangsaan Malaysia, Jalan Raja Muda Abdul Aziz, Kuala Lumpur, Malaysia
Correspondence: Bariah Mohd-Ali Optometry and Vision Science Program, School of Healthcare Sciences, Faculty of Health Science, Universiti Kebangsaan Malaysia, Jalan Raja Muda Abdul Aziz, 50300 Kuala Lumpur, Malaysia

Email bariahli@yahoo.com
Background: Rigid gas permeable (RGP) and silicone hydrogel (SH) contact lenses with specific designs are currently being used to improve visual function in patients with keratoconus. However, there are minimal data available comparing the effects of these lenses on visual function in patients with keratoconus. The objectives of this study were to compare visual acuity and contrast sensitivity using spectacles, RGP lenses, and SH lenses, and to evaluate the effects of RGP and SH lenses on higher-order aberrations and visual quality in eyes with keratoconus. The relationship between visual outcomes, aberration, and visual quality were also examined.

Methods: This was a pilot study involving 13 eyes from nine subjects with keratoconus. Subjects were fitted with RGP and SH contact lenses. Visual acuity and contrast sensitivity were measured using Snellen and Pelli-Robson charts, respectively. Ocular aberrations and visual quality were measured using an OPD-Scan II device. All measurements were conducted before and after contact lens wear.

Results: Significantly better visual acuity was obtained with RGP lenses than with spectacles or SH lenses $(P<0.001)$. No significant difference in contrast sensitivity values was detected between RGP and SH lenses $(P=0.06)$. Both SH and RGP lenses significantly reduced total ocular and higher-order aberrations $(P<0.001)$ when compared with spectacles, but RGP lenses reduced trefoil, coma, and spherical aberrations more than $\mathrm{SH}$ lenses. No significant difference in astigmatic aberrations was found between RGP and SH lenses $(P=0.12)$. Negative correlations were found between visual acuity and coma aberration and contrast sensitivity with higherorder aberrations and coma, trefoil, and astigmatic aberrations. Regarding visual quality, no significant difference was found between RGP and SH lenses, but RGP significantly improved visual quality when compared with spectacles $(P=0.04)$.

Conclusion: RGP lenses provided better visual acuity and greater reduction of aberrations than spectacles or SH lenses in patients with keratoconus. However, more data are needed to confirm these findings.

Keywords: visual acuity, contrast sensitivity, aberration, visual quality, keratoconus

\section{Introduction}

Keratoconus is a fairly common bilateral, noninflammatory, degenerative, axial, ectatic condition of the cornea in which the cornea assumes an irregular conical shape. ${ }^{1}$ Because the cornea acts as the most powerful optical system in the eye, the morphological corneal alterations (thinning and protrusion) associated with keratoconus are accompanied by optical defects or aberrations. ${ }^{2}$ Reduction in best-corrected visual acuity has been reported in keratoconus due to disease progression. In the Collaborative Longitudinal Evaluation of Keratoconus group (CLEK) study, subjects with keratoconus showed a small but clear reduction in best-corrected visual acuity 
during follow-up, with low-contrast acuity deteriorating more rapidly than high-contrast acuity. ${ }^{3}$

Corneal distortion produces irregular higher corneal astigmatism, which is the most frequent cause of increased aberrations and reduced optical quality in keratoconus. ${ }^{4,5}$ Thus, the increase in higher-order ocular (total) aberrations in keratoconus results from increased higher-order corneal aberrations. ${ }^{4}$ Compared with normal eyes, eyes with or suspicious for keratoconus had significantly larger higher-order aberrations and third-order (coma) aberration. ${ }^{6}$ Characterizing the aberrations in keratoconus, Pantanelli et $\mathrm{al}^{7}$ found that keratoconic eyes had higher-order aberrations that were approximately 5.5 times more than what is typical in normal eyes. High-order aberrations, and coma aberrations in particular, have been found to decrease visual acuity, ${ }^{8,9}$ low-contrast visual acuity, ${ }^{8}$ and contrast sensitivity. ${ }^{10}$

Contact lenses are the treatment of choice for most patients with keratoconus. They provide a regular refractive surface, thus eliminating much irregular astigmatism, and allow better vision. ${ }^{11}$ Many studies have estimated that at least three quarters of patients with keratoconus in the developed world can be treated successfully using a number of types of contact lenses. ${ }^{12}$ A review of the literature shows a reduction in magnitude of higher-order aberrations in keratoconic eyes with the wearing of rigid gas permeable (RGP) contact lenses. ${ }^{2,13,14}$ When applied to the eye, the RGP lens can mask all corneal aberrations by replacing the irregular cornea with the regular and smooth refractive surface of the RGP and the tear lens. ${ }^{15}$

Due to the hard RGP materials and the association between keratoconus and a number of irritating systemic or ocular conditions such as atopy, ${ }^{16}$ many patients with keratoconus experience discomfort that may lead to intolerance of RGP lenses. ${ }^{17,18}$ There are several reports describing the use of soft contact lenses as an alternative to RGP lenses. ${ }^{19,20}$ However, few soft contact lenses designed for correction of keratoconus are currently available. Gonzales-Meijome et $\mathrm{al}^{21}$ reported successful rehabilitation of three keratoconic eyes using the Soft $\mathrm{K}$ lens, with visual acuity of 6/4.5 being achieved. Yamazaki et al ${ }^{22}$ fitted 80 keratoconic eyes with spherical and spherocylindrical soft contact lenses and found that over $90 \%$ of eyes achieved vision of $6 / 12$ or better. These studies indicate that soft hydrogel lenses may be useful in patients who cannot tolerate RGP lenses. Recently, White ${ }^{23}$ described the use of soft silicone hydrogel ( $\mathrm{SH}$ ) lenses designed to improve oxygen permeability in conical corneas. These lenses were manufactured with a slightly thicker center $(0.4 \mathrm{~mm})$ in order to mask corneal astigmatism.
In a study of 13 keratoconic patients, Griffiths et $\mathrm{al}^{15}$ compared the visual performance of RGP lenses with that of four different spherical soft lenses. They found that RGP lenses gave better high-contrast (by approximately one line) and low-contrast (by approximately 1.5 lines) $\log$ MAR (logarithm of the minimum angle of resolution) acuity. The authors also reported that RGP lenses significantly reduced residual corneal aberrations, at diameters of $4 \mathrm{~mm}$ and $6 \mathrm{~mm}$, compared with all four soft lenses evaluated $(P<0.01)$. Jinabhai et $\mathrm{al}^{24}$ found that RGP lenses provided superior visual quality and greater reduction of third-order aberrations when compared with toric soft contact lenses in a group of patients with keratoconus.

RGP and SH lens designs are currently used to improve visual outcomes in patients with keratoconus. However, relatively few studies have compared the effects of $\mathrm{SH}$ lenses on visual performance and optical aberrations with those of RGP lenses and spectacles. This was a pilot study in Malaysia that investigated and compared changes in visual acuity and contrast sensitivity in patients with keratoconus using SH lenses, RGP contact lenses, or spectacle correction. The study also evaluated the effects of RGP and SH lenses on higher-order aberrations and optical quality in patients. The relationship between visual outcome and aberration was also examined.

\section{Materials and methods Subjects}

Thirteen eyes of nine patients recently diagnosed with keratoconus and referred from hospitals and optometry clinics from around Kuala Lumpur were invited to participate in this study. To be eligible for participation, subjects had to meet certain inclusion criteria, ie, existence of keratoconus detected by slit-lamp biomicroscopy (stromal thinning, Vogt's striae, Fleischer rings) or by OPD-Scan classification map, and ability to be fitted with RGP and SH contact lenses. Eyes with corneal scarring or any ocular disorder other than keratoconus and a history of ocular surgery were excluded.

The study followed the tenets of the Declaration of Helsinki. All participants gave their informed consent after being told about the purpose of the study. The investigation protocol was approved by the medical ethics committee at the Universiti Kebangsaan Malaysia.

\section{Visual performance}

Refractive error was measured with a retinoscope (Neitz Instruments Co, Ltd, Tokyo, Japan). Retinoscopy was performed in a dark room using a fixation target at 6 meters. 
The results were refined subjectively using a Snellen chart (Haag-Streit UK, Harlow, UK) and a trial set of lenses. The Snellen chart was used to evaluate distance visual acuity at 6 meters. Subjects were seated and their visual axis was aligned with the center of the chart using an adjustable chair. Subjects were required to attempt to read all letters from top to bottom on the chart until he/she missed three or more letters in a single line after attempting all letters on that line, and a visual acuity score was recorded accordingly. Visual acuity was taken for each eye without optical correction (unaided), with spectacles, and with the prescribed RGP and $\mathrm{SH}$ lenses. The test illumination level was approximately 480-600 lux.

Contrast sensitivity was measured using the Pelli-Robson test chart. This chart is approximately $59 \times 84 \mathrm{~cm}$ and is printed on rigid cardboard. It consists of 48 letters arranged in eight rows of six letters each. Each line consists of two triplets. Each triplet contains letters of equal contrast, and the contrast of each triplet decreases by a factor of $0.15 \mathrm{log}$ contrast sensitivity units. The stated contrast varies from $100 \%$ (0.00 log contrast sensitivity units) to $0.56 \%(-2.25 \mathrm{log}$ contrast sensitivity units). Each letter subtends 2.8 degrees at a test distance of one meter. ${ }^{25}$ Measurement of contrast sensitivity was conducted at a working distance of one meter under room illumination levels of approximately 480-600 lux as recommended by the manufacturers. The subject's eye was at the same level as the center of the test chart. The subjects were then instructed to read all letters on the chart beginning with the highest contrast letters. Subjects were allowed up to 30 seconds to read a single letter if needed and forced to guess until the subject missed two of three letters in a triplet. Evaluation of contrast sensitivity was carried out at the baseline visit with spectacle correction and with the prescribed contact lens.

\section{Corneal slit-lamp examination and contact lens fitting assessment}

Corneal assessment was carried out using a slit-lamp biomicroscope (SL-1E, Topcon Medical Systems Inc., Tokyo, Japan). The device was used in all stages of the study (including patient selection and assessment of contact lens fitting). At baseline, the slit-lamp biomicroscope was used to detect signs of keratoconus, including stromal thinning, Vogt's striae, Fleischer's ring, and Munson's sign.

The slit-lamp biomicroscope was also used to assess fitting of the selected contact lens. RGP contact lenses with RoseK and RoseK2 IC lens designs (Menicon Co, Ltd, Nagoya, Japan) and SH contact lenses with a Kerasoft IC design (Ultravision CLPL, Leighton Buzzard, UK) were used in this study. All fittings were conducted following the guidelines set by the manufacturer.

\section{Corneal topography and ocular aberrations}

Corneal topography was assessed using an optical path difference scanning system (OPD-Scan II, Nidek Co, Ltd, Gamagori, Japan). The OPD-Scan II provides information on corneal topography, wavefront, autorefraction, keratometry, and pupillometry in one unit. The corneal topography function utilizes Placido disc technology in which the image of reflected rings of light from the cornea is captured. Thousands of data points are analyzed to plot corneal contour, shape, and refractive power. ${ }^{26}$ The OPD-Scan II offers several data maps providing information on corneal shape (axial and refractive corneal maps). A corneal classifier map was used at the baseline visit to confirm the diagnosis. Sim K1 and Sim K2 for each keratoconic eye and corneal astigmatism were monitored and recorded with spectacle correction, SH lenses, and RGP contact lenses.

Ocular aberrations and higher-order corneal aberrations were measured from the total wavefront and wavefront high-order aberration OPD maps using the root mean square (RMS). Wavefront aberrations were computed up to the sixth Zernike order for a $5 \mathrm{~mm}$ pupil diameter. The Zernike polynomial graph plots the magnitude of each of the aberration components, including spherical aberration, coma, and trefoil, that make up the total aberration of the eye. An automated eye image was captured for each eye, and the different aberration maps were then evaluated using the device's internal software (OPD-Station version 2.13 [CN 1.02]).

Total maps of Zernike polynomials included sixth order decomposition (excluding the piston and lower-order terms). The aberration groups evaluated were total ocular aberration (included lower-order and higher-order aberration terms), total higher-order aberration (all terms included in the third, fourth, fifth, and sixth order), total coma (coma terms from third to fifth order), total trefoil (trefoil terms in third to fifth order), total spherical aberration (spherical aberration terms in fourth and sixth order), total tetrafoil (tetrafoil terms in fourth and sixth order) and higher-order astigmatism (astigmatism terms in fourth and sixth order).

The OPD-Scan II also measures the Strehl ratio, which is used to evaluate optical image formation and is useful for estimating the visual quality of eyes with aberration. Values for total ocular aberrations, higher-order aberrations, Zernike polynomial terms until sixth order, and quality of vision 
(Strehl) ratio were obtained for each eye without optical correction, and with SH and RGP contact lenses.

\section{OPD-Scan II validation}

Several studies have confirmed the reliability and repeatability of the OPD-Scan II machine. Hieda and Kinoshita ${ }^{27}$ compared ocular aberration using the OPD-Scan II and the HartmannShack wavefront sensor in both model eyes and human eyes. Although the two devices use different approaches to determine aberrations, the aberration values using the OPD-Scan II were found to be similar to those for the Hartmann-Shack device, especially for large pupils. Bartsch et $\mathrm{al}^{28}$ compared the aberration results for the Tracey visual function analyzer and the OPD-Scan II in 38 healthy eyes, and reported that both devices showed similar reproducibility. The OPD-Scan II used in the current study was subjected to a validation test, where measurements were taken for ten nonkeratoconic subjects (20 eyes) on two consecutive mornings. The correlation coefficients $(r)$ between the two different readings were found to be $0.98,0.99$, and 0.94 for mean $\mathrm{K}$ readings, corneal astigmatism, and total aberration, respectively, with a significance level of $P<0.001$.

\section{Data collection}

All subjects underwent a full ophthalmic examination at the baseline visit. These examinations included visual acuity and contrast sensitivity, subjective and objective refraction, slit-lamp biomicroscopic examination (to reveal signs of keratoconus), and corneal topography (OPD-Scan II). Visual acuity and contrast sensitivity were evaluated with each subject's optimum spectacle correction. The subjects were then fitted with suitable RGP (Rose K2 or Rose K2 IC) lenses, and optimal lens parameters were ordered. Subjects who were contact lens wearers were told to stop wearing their usual contact lenses for 4 weeks as a washout period and then to come for an initial examination and RGP lens fitting. On the delivery date, RGP lens fitting was checked again and similar baseline examinations were carried after 2 hours (with the contact lens worn) to ensure the subject's adaptation to the contact lens. Subjects were then given another appointment on the next day and a similar procedure was carried out using the SH contact lens.

\section{Data analysis}

Total ocular aberrations, higher-order aberrations, and total coma, total trefoil, total spherical, and total higher-order astigmatism were detected from OPD-Scan II wavefront and polynomial Zernike maps. Statistical Package for the
Social Sciences version 20 software (IBM Corporation, Armonk, NY, USA) was used to analyze the data. Normality of the data was checked using the Shapiro-Wilk test with a critical value of 0.05 . Normally distributed data were analyzed using one-way repeated-measures analysis of variance, and Huynh-Feldt correction was used in the event of violation of sphericity assumption. Non-normally distributed data were evaluated using the Friedman test. Further post hoc analyses were carried out for normally distributed data. For non-normally distributed data, post hoc tests were carried out using the Wilcoxon signed-ranks test (WSRT). Correlations were carried using Pearson's bivariate test. A statistical significance level of $P<0.05$ was chosen as the critical value for all statistical tests used in this study.

\section{Results \\ Subject demographics}

The subjects comprised six males and three females of mean age 24.22 \pm 6.59 (range 15-33) years. Table 1 summarizes the corneal surface keratometric readings, spherical equivalent of refractive errors, corneal slit-lamp appearance, and $\mathrm{CLEK}^{29}$ severity for the keratoconic eyes at baseline. Five of the eyes studied were graded as having severe keratoconus, seven eyes were graded as having moderate keratoconus, and one eye was graded as having mild keratoconus. Slit-lamp biomicroscopy showed that the most common sign of keratoconus was Vogt's striae, seen in ten eyes. At baseline, the mean spherical refractive error was $-1.98 \pm 2.44 \mathrm{D}$,

Table I Corneal surface keratometry, SE, corneal slit lamp signs, and CLEK study group severity scale for the study subjects at the initial examinations

\begin{tabular}{llllll}
\hline Patient & SE & Average K & Steep K & $\begin{array}{l}\text { Vogt's } \\
\text { striae }\end{array}$ & $\begin{array}{l}\text { CLEK } \\
\text { grade }\end{array}$ \\
\hline I & -7.50 & 47.13 & 53.15 & Absent & Severe \\
2 & -3.75 & 56.94 & 61.25 & Present & Severe \\
3 & -9.00 & 64.70 & 71.81 & Present & Severe \\
4 & -0.75 & 44.88 & 46.94 & Present & Moderate \\
5 & -5.50 & 54.97 & 56.53 & Present & Severe \\
6 & -1.50 & 46.18 & 47.94 & Present & Moderate \\
7 & -1.50 & 44.83 & 45.24 & Absent & Moderate \\
8 & -2.75 & 43.22 & 43.49 & Absent & Mild \\
9 & -5.25 & 47.66 & 49.27 & Present & Moderate \\
I0 & -10.50 & 53.82 & 55.88 & Present & Severe \\
II & -5.25 & 48.75 & 50.37 & Present & Moderate \\
I2 & -3.00 & 45.59 & 50.07 & Present & Moderate \\
I3 & -2.50 & 44.66 & 48.35 & Present & Moderate \\
\hline
\end{tabular}

Note: SE, average K, and steep $\mathrm{K}$ were in diopters. $\mathrm{K}$ represents keratometry reading. Abbreviations: CLEK, Collaborative Longitudinal Evaluation of Keratoconus; SE, spherical equivalent of refraction. 
the mean astigmatic refractive error was $-4.80 \pm 4.24 \mathrm{D}$, and the mean spherical equivalent was $-4.38 \pm 3.07 \mathrm{D}$.

\section{Visual performance}

Table 2 summarizes the mean values for visual acuity and contrast sensitivity measured using the three correction methods. Nonparametric analysis showed that visual acuity with the RGP contact lens was significantly better than with spectacles (WSRT, $\mathrm{z}=-3.18, P=0.001$ ) and with $\mathrm{SH}$ contact lenses (WSRT, $\mathrm{z}=-3.21, P=0.001$ ). However, no significant difference in visual acuity was found between SH lenses and spectacle correction (WSRT, $\mathrm{z}=-1.75, P=0.08$ ). A significantly higher contrast sensitivity value was obtained with the RGP contact lenses than with spectacles (WSRT, $\mathrm{z}=-2.55$, $P=0.01)$. However, no statistically significant difference was found between SH contact lenses and spectacles (WSRT, $\mathrm{z}=-1.65, P=0.09$ ).

Analysis of visual quality (Strehl ratio) using the Friedman test showed a significant difference between measures taken with no optical correction, with SH contact lenses, and with RGP contact lenses, with mean ranks of 1.5, 1.81, and 2.69 , respectively $\left(\chi^{2}=10.16, P=0.006\right)$. Visual quality with RGP contact lenses $(0.019 \pm 0.01)$ was found to be significantly better than that before contact lens wear $(0.016 \pm 0.42$; WSRT, $\mathrm{z}=-1.85, P<0.04)$. No significant difference was found between the RGP and SH lenses $(0.017 \pm 0.03)$ or between $\mathrm{SH}$ lenses and measurements taken without optical correction ( $P=0.15$ and $P=0.19$, respectively).

\section{Ocular aberrations}

Total ocular aberrations, higher-order aberrations, and polynomial Zernike values were compared before and after wearing RGP and SH contact lenses (Figure 1). Significant differences in total ocular aberrations and higher-order aberrations were noted between the groups $(P<0.001)$. Further analysis showed significantly lower total ocular aberrations and higher-order aberrations with RGP lenses when

Table 2 Best-corrected visual acuity and contrast sensitivity measured with spectacles, SH lenses, and RGP lenses

\begin{tabular}{llll}
\hline Visual function & Spectacles & SH lenses & RGP lenses \\
\hline BCVA (decimal) & & & \\
$\quad$ Mean \pm SD & $0.56 \pm 0.26$ & $0.69 \pm 0.27$ & $1.12 \pm 0.15$ \\
$\quad$ Range & $0.17-1.00$ & $0.25-1.00$ & $0.70-1.20$ \\
CS (Log CS) & & & \\
$\quad$ Mean \pm SD & $1.65 \pm 0.24$ & $1.78 \pm 0.22$ & $1.86 \pm 0.14$ \\
$\quad$ Range & $1.20-1.95$ & $1.20-1.95$ & $1.65-1.95$ \\
\hline
\end{tabular}

Abbreviations: BCVA, best-corrected visual acuity; CS, contrast sensitivity; RGP rigid gas permeable; $\mathrm{SH}$, silicone hydrogel; SD, standard deviation. compared with SH contact lenses $(P=0.001)$. Descriptive statistics indicated that total ocular aberration and higherorder aberration values were decreased by $80.55 \%$ and $71.11 \%$, respectively, with RGP lens wear versus $45.9 \%$ and $38.10 \%$ with SH lens wear. Repeated-measures analysis of variance indicated significant differences between the three groups of measurements for total trefoil RMS $(P<0.001)$. Pairwise analysis indicated that total trefoil RMS values were significantly lower for RGP lenses $(0.42 \pm 0.25)$ when compared with measurements taken without contact lenses (1.85 \pm 1.23$)$. A significantly lower total trefoil RMS was also found with the SH lens $(0.93 \pm 0.89)$ when compared with measurements taken without contact lenses. However, no significant difference in total trefoil RMS values $(P=0.14)$ was found between the RGP and SH lenses.

Statistical analysis also indicated a significant difference in total coma aberrations between the three groups of measurements $(P=0.002)$. Pairwise comparisons showed significant lower total coma aberrations for measurements with RGP lenses $(0.51 \pm 0.24)$ compared with those taken without contact lenses (1.85 \pm 1.49$)$. Total coma RMS did not show significant differences between $\mathrm{SH}$ lenses and nonuse of contact lenses (1.28 \pm 1.10$)$.

Friedman nonparametric analysis did not show any significant differences between the three groups of measures in term of total spherical aberrations $(P=0.19)$. Further nonparametric tests did not show any significant differences between total spherical aberrations measured with RGP lenses and those measured without wearing contact lenses $(P=0.25)$. The analysis also indicated that RGP contact lenses significantly lowered total spherical aberration values when compared with SH lenses $(P=0.03)$.

With regard to astigmatic aberration, results from the nonparametric analysis showed significant differences between the three readings $(P=0.03)$. The RGP contact lens was found to reduce total astigmatic aberrations significantly more than without correction $(P=0.02)$. However, no significant differences were detected between the SH and RGP lenses $(P=0.15)$ or between $\mathrm{SH}$ lenses and no use of contact lenses $(P=0.12)$.

Repeated-measures analysis of variance showed no significant difference (Approach significant) between the three groups of measurements in term of total tetrafoil RMS $(P=0.07)$. Pairwise comparisons showed no significant difference between the RGP lens $(0.24 \pm 0.25)$ and the measures taken without contact lenses $(P=0.06)$. Total tetrafoil RMS did not show a significant difference between measurements taken with SH lenses $(0.44 \pm 0.58)$ and RGP lenses or 


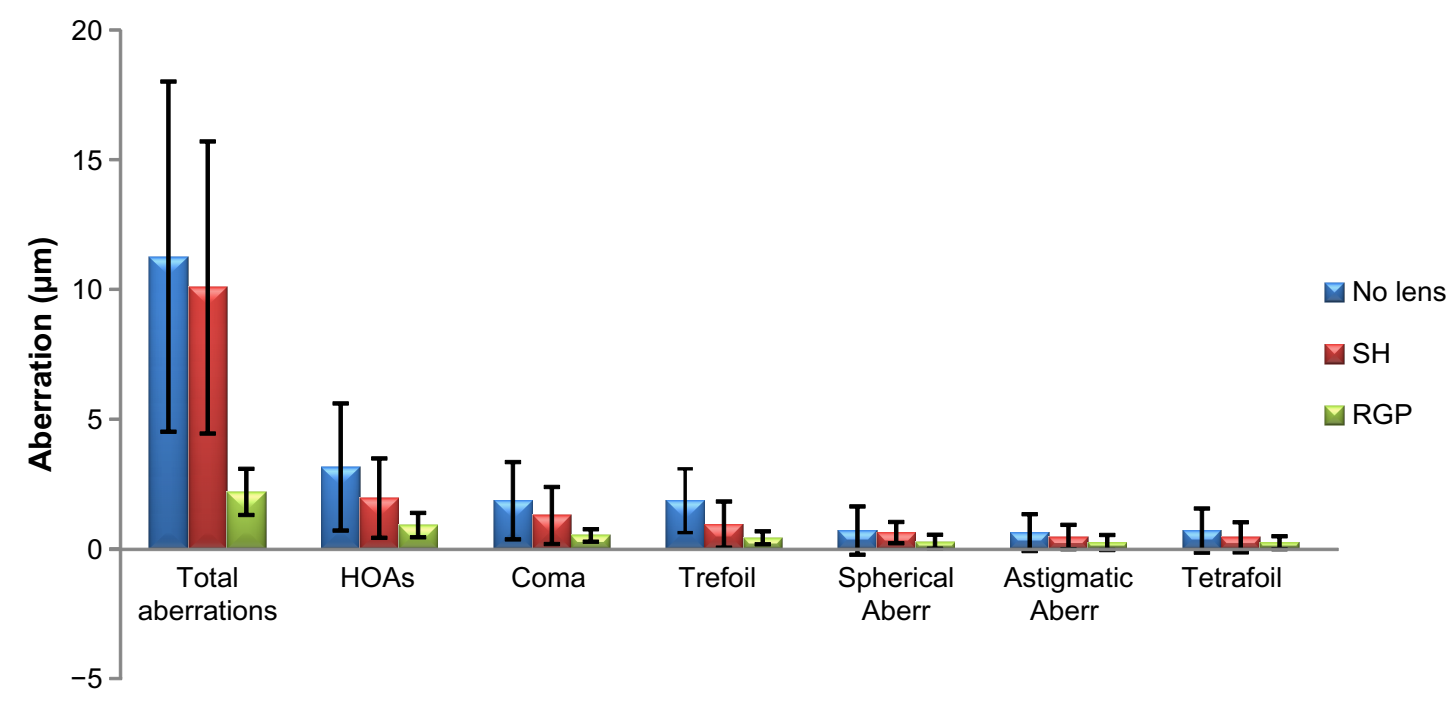

Figure I Ocular aberrations measured with no lenses, with RGP and SH lenses.

Notes: Values are reported as the mean \pm standard deviation in $\mu \mathrm{m}$.

Abbreviations: RGP, rigid gas permeable; $\mathrm{SH}$, silicone hydrogel; HOAs, higher-order aberrations.

between SH lenses and nonuse of contact lenses $(0.71 \pm 0.88)$. Table 3 shows the post hoc results for all types of ocular aberration evaluated and the three different methods used for correction.

\section{Corneal topography}

Table 4 shows the means for corneal topographic parameters measured with no lenses, with RGP lenses, and SH lenses. One-way repeated-measures analysis of variance showed significant differences between the three conditions in term of the steepest keratometric reading and the average keratometric reading $(P=0.001$ for both). A series of pairwise

Table 3 Post hoc data from comparing ocular aberrations and corneal parameters measured with no lenses, RGP lenses, and $\mathrm{SH}$ lenses

\begin{tabular}{llll}
\hline & $\begin{array}{l}\text { No lens } \\
\text { versus RGP }\end{array}$ & $\begin{array}{l}\text { No lens } \\
\text { versus SH }\end{array}$ & $\begin{array}{l}\text { RGP } \\
\text { versus SH }\end{array}$ \\
\hline $\begin{array}{l}\text { Ocular aberrations } \\
\text { Total aberrations }\end{array}$ & $0.00 I^{*}$ & $0.003^{*}$ & $0.00 I^{*}$ \\
HOAs & $0.017^{*}$ & $0.013^{*}$ & 0.091 \\
T coma & $0.015^{*}$ & 0.25 & 0.064 \\
T trefoil & $0.003^{*}$ & $0.005^{*}$ & 0.14 \\
T spherical aberration & 0.25 & 0.97 & $0.03^{* *}$ \\
T astigmatic aberration & $0.02^{* *}$ & 0.12 & 0.15 \\
T tetrafoil & 0.06 & 0.10 & 0.27 \\
Corneal parameters & & & \\
Steepest K readings & $0.004^{*}$ & $0.005^{*}$ & $0.028^{*}$ \\
Average K readings & $0.007^{*}$ & $0.005^{*}$ & 0.38 \\
Corneal astigmatism & $0.002^{*}$ & 0.45 & $0.00 I^{*}$ \\
\hline
\end{tabular}

Notes: *Significant difference using repeated-measures analysis of variance and post hoc; **significant difference using WSRT analysis.

Abbreviations: HOAs, higher-order aberrations; RGP, rigid gas permeable; SH, silicone hydrogel; WSRT, Wilcoxon signed-ranks test; K, keratometry; T, total. comparisons showed that average $\mathrm{K}$ values measured with wear of RGP lenses (42.16 \pm 1.74$)$ and SH lenses (43.31 \pm 2.45$)$ were significantly lower than without lenses $(49.49 \pm 6.30)$. No significant differences in average $\mathrm{K}$ readings were found between the RGP and SH lenses $(P=0.38)$. However, differences were detected in term of the steepest $\mathrm{K}$ readings $(P=0.028)$ between the two lenses.

Statistical analysis indicated significant differences in corneal astigmatism between the three methods of correction $(P<0.001)$. Pairwise comparisons showed that corneal astigmatism with RGP lenses $(0.94 \pm 0.76)$ was significantly reduced when compared with no use of contact lenses $(-6.37 \pm 4.48, P=0.002)$ and with SH lenses $(-5.98 \pm 4.00$, $P=0.001)$. No significant difference in corneal astigmatism was noted between SH lenses and no use of contact lenses $(P=0.45)$.

\section{Correlation analysis}

\section{Visual performance and corneal topography}

Visual acuity and contrast sensitivity measured with spectacles, RGP lenses, and SH lenses were plotted against the steepest $\mathrm{K}$ readings, average $\mathrm{K}$ readings, and corneal astigmatism. The analysis showed no significant correlation between visual acuity and steepest $\mathrm{K}$ reading measurements for any of the three methods of correction $(P>0.05)$. A significant moderate negative correlation was found between the steepest $\mathrm{K}$ reading and contrast sensitivity measured with spectacle correction at baseline $(r=-0.64$, $P=0.02$, Figure $2 \mathrm{~A}$ ). The analysis also showed that visual acuity was not correlated with average $\mathrm{K}$ readings in any 
Table 4 Comparing corneal parameter means between no lens, with SH and with RGP lenses and repeated-measures analysis of variance

\begin{tabular}{lllll}
\hline Corneal parameters & No lens & SH & RGP & Statistics/P-value \\
\hline Steepest K reading & $53.02 \pm 8.42$ & $46.3 I \pm 3.97$ & $42.67 \pm I .84$ & $\mathrm{~F}_{(1.2,14.6)}=15.98 / P=0.00 I^{*}$ \\
Average K reading & $49.49 \pm 6.30$ & $43.3 \mathrm{I} \pm 2.45$ & $42.16 \pm I .74$ & $\mathrm{~F}_{(1.2,14.61)}=\mid 14.12 / P=0.00 I^{*}$ \\
Corneal astigmatism & $-6.37 \pm 4.48$ & $-5.98 \pm 4.00$ & $-0.94 \pm 0.76$ & $\mathrm{~F}_{(1.2,14.61)}=\mid 4.12 / P=0.00 I^{*}$ \\
\hline
\end{tabular}

Notes: Values are reported as the mean \pm standard deviation in $\mu \mathrm{m}$; *significant value.

Abbreviations: RGP, rigid gas permeable; $\mathrm{SH}$, silicone hydrogel; K, keratometry.

of the study groups. However, a significant strong negative correlation was noted between contrast sensitivity and average $\mathrm{K}$ readings with spectacle correction $(r=-0.69, P=0.008$, Figure 2B). No significant correlation was found between visual performance and corneal astigmatism $(P>0.05)$ for the three methods of correction.

\section{Visual performance and ocular aberrations}

Visual acuity and contrast sensitivity measured using each correction mode (spectacles, SH lens, and RGP lens) were plotted against the aberration terms evaluated. Spectacle visual acuity was found not to be related to changes in total ocular aberrations, higher-order aberrations, total coma RMS, total trefoil RMS, or total spherical aberrations.
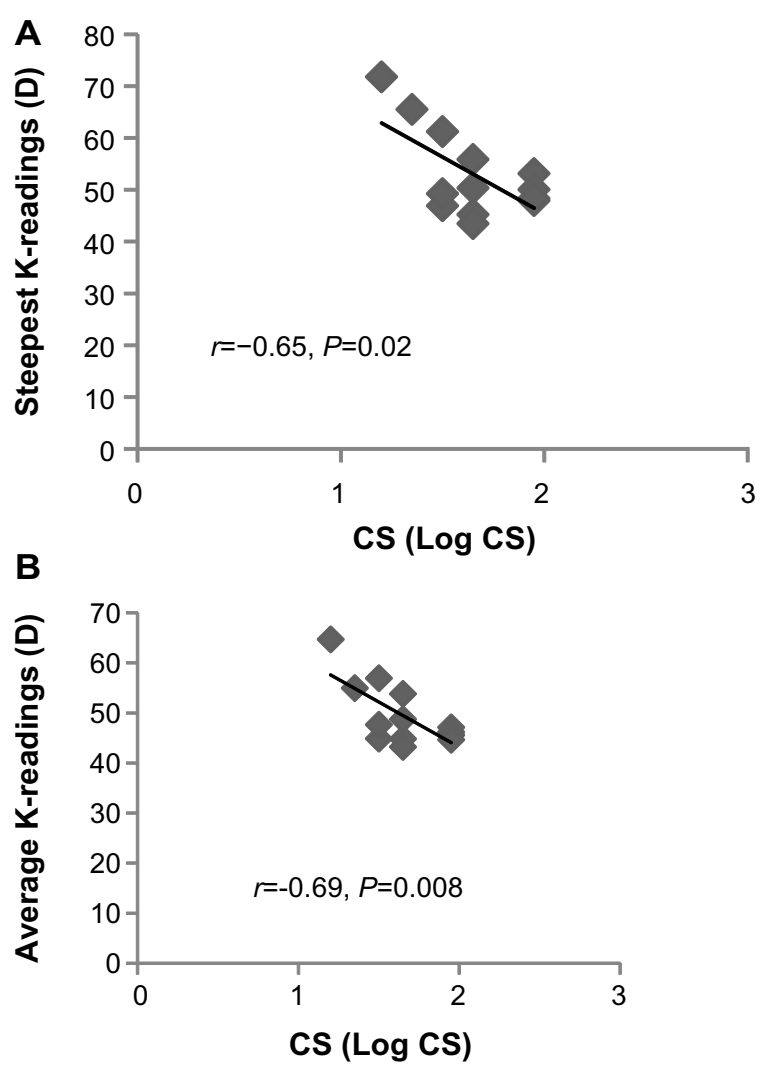

Figure 2 Pearson's correlation between contrast sensitivity and (A) steepest K readings and $(\mathbf{B})$ average $K$ readings measured with spectacle correction at baseline. Abbreviations: CS, contrast sensitivity; D, diopter; K, keratometry.
However, a significant correlation was found between total astigmatic aberrations $(r=-0.57, P=0.04$, Figure $3 \mathrm{~A}$ ) and total tetrafoil RMS ( $r=-0.74, P=0.002$, Figure $3 \mathrm{~B})$. Spectacle contrast sensitivity was found to be significantly correlated with higher-order aberrations ( $r=-0.56, P=0.04)$, total spherical aberration $(r=-0.67, P=0.01)$, the total astigmatic component of aberration $(r=-0.63, P=0.02)$, and total tetrafoil aberration $(r=-0.55, P=0.04$, Figure 4A-D). No correlation was found between spectacle contrast sensitivity and total ocular aberration, total coma, or total trefoil aberrations $(P>0.05)$.

Statistical analysis showed that visual acuity with the RGP lenses was not correlated with any of the aberration terms measured $(P>0.05)$. However, contrast sensitivity with the RGP lenses was correlated with total trefoil aberrations ( $r=-0.71, P=0.007$ ). Visual acuity values measured with $\mathrm{SH}$ lenses were not correlated with total ocular aberrations, higher-order aberrations, total trefoil RMS, total spherical, total astigmatic, or total tetrafoil aberrations $(P>0.05)$, but a significant negative correlation with total coma RMS $(r=-0.56, P=0.04$, Figure 5A) was detected. The analysis also showed that contrast sensitivity measurements with the SH lenses had a significant linear correlation with total coma RMS ( $r=-0.60, P=0.02$, Figure 5B), higher-order aberrations $(r=-0.69, P=0.009$, Figure $6 \mathrm{~A})$, total trefoil $(r=-0.72, P=0.006$, Figure 6B), and total astigmatic aberrations ( $r=-0.76, P=0.003$, Figure $6 \mathrm{C}$ ), and had a strong negative correlation with total tetrafoil aberrations $(r=-0.81$, $P=0.001$, Figure 6D). However, no correlation was detected with total RMS and total spherical RMS.

Pearson's correlation was also used to determine the relationship between the quality of vision indicator (Strehl ratio; with no use of contact lenses, with RGP lenses, and with SH lenses) and visual performance (visual acuity and contrast sensitivity). This test found no significant differences $(P>0.05)$.

\section{Discussion}

This pilot study investigated the effects of RGP lenses, SH lenses, and spectacles on visual performance (visual acuity and contrast sensitivity) in a sample of patients with 
A

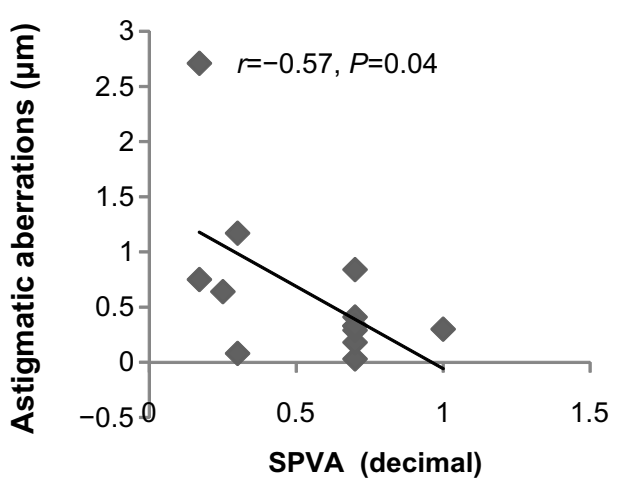

B

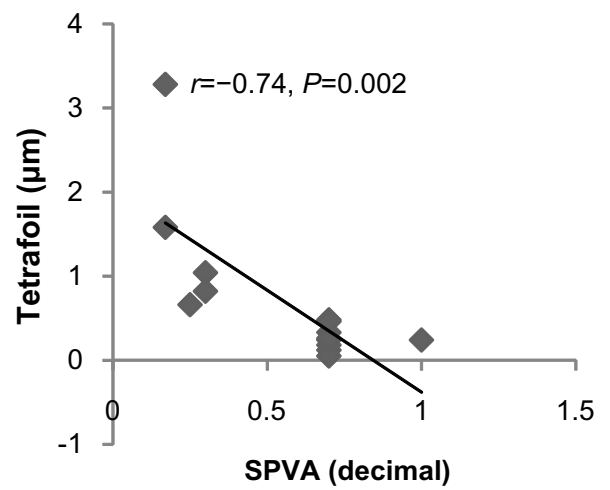

Figure 3 Correlation between SPVA and (A) astigmatic aberration and (B) total tetrafoil RMS. Abbreviations: SPVA, spectacle visual acuity; RMS, root mean square.

A

$r=-0.56, P=0.04$

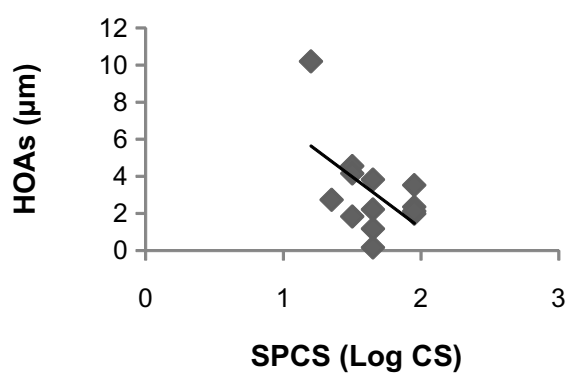

C

$r=-0.63, P=0.02$

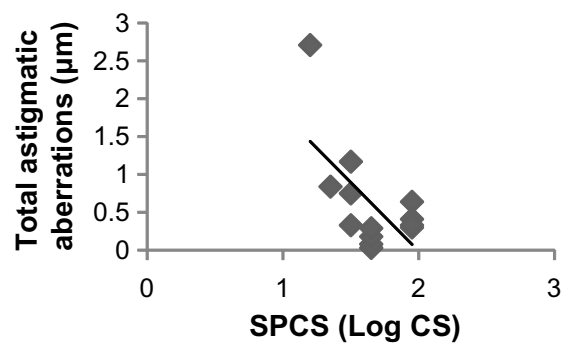

B

$r=-0.67, P=0.01$

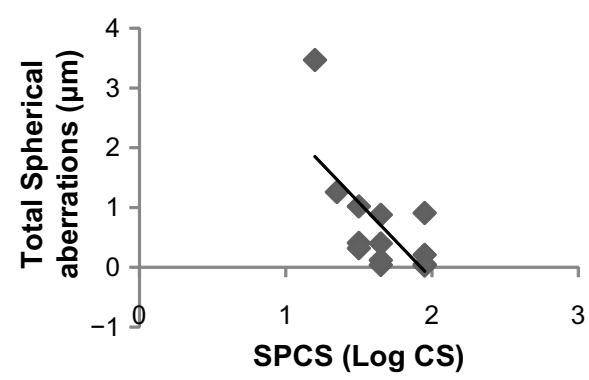

D

$r=-0.55, P=0.04$

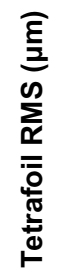

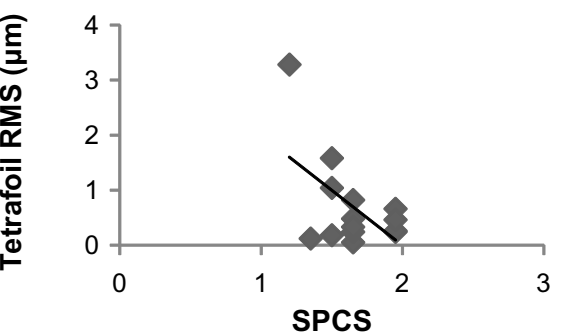

Figure 4 Correlations between spectacle contrast sensitivity and (A) HOA RMS, (B) total spherical aberrations, (C) total astigmatic aberrations, and (D) total tetrafoil RMS. Abbreviations: CS, contrast sensitivity; SPCS, spectacle contrast sensitivity; HOA, higher-order aberration; RMS, root mean square.

A

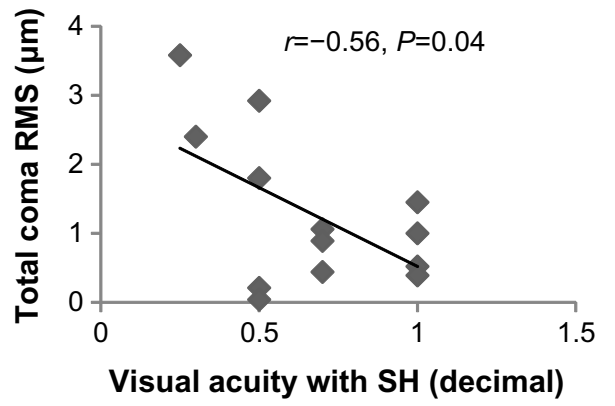

B

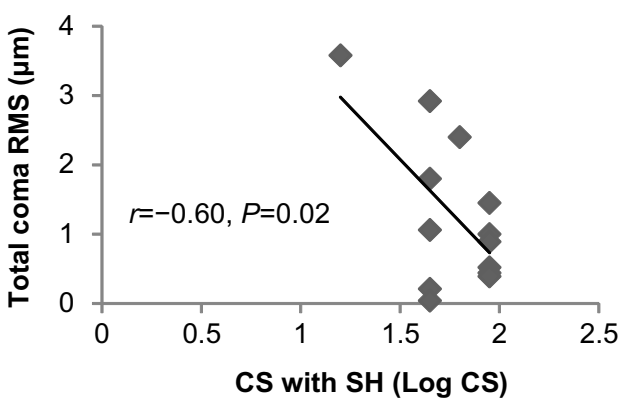

Figure 5 Correlations between total coma RMS and (A) visual acuity with SH and (B) contrast sensitivity with SH. Abbreviations: $\mathrm{SH}$, silicone hydrogel; RMS, root mean square; CS, contrast sensitivity. 
A

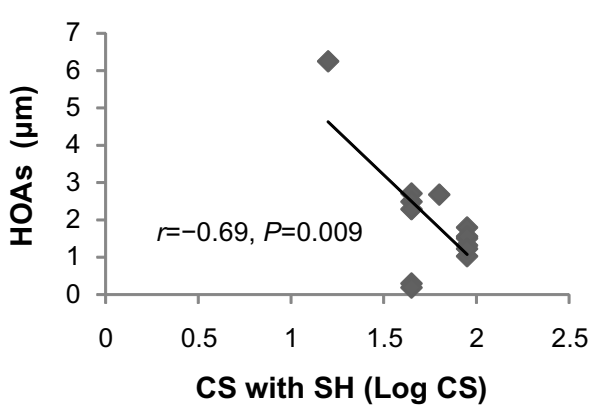

C

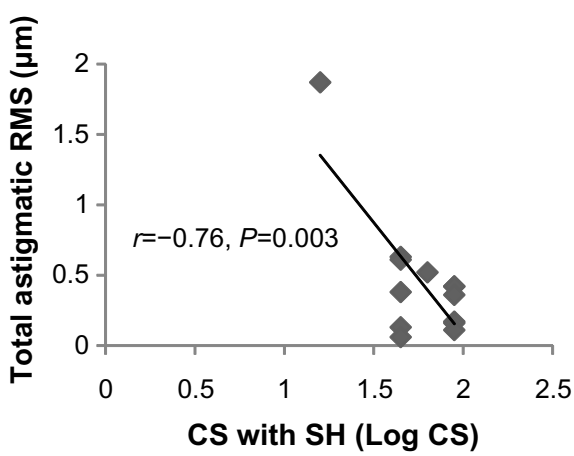

B

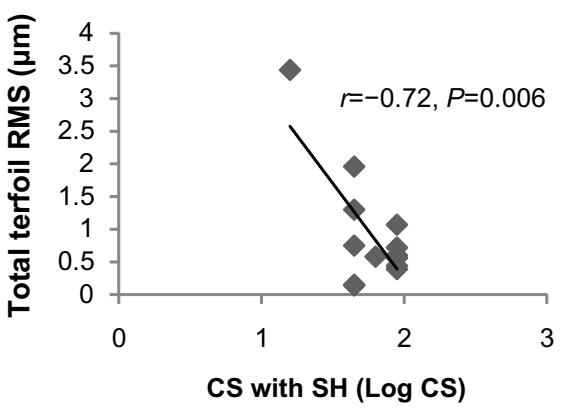

D

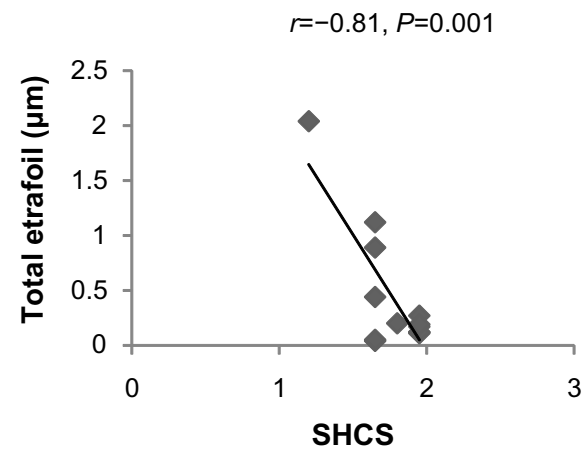

Figure 6 Correlations between CS measured with SH lenses and (A) HOA, (B) total trefoil RMS, (C) total astigmatic aberrations and (D) total tetrafoil RMS. Abbreviations: CS, contrast sensitivity; HOA, higher-order aberration; SH, silicone hydrogel; RMS, root mean square; SHCS, contrast sensitivity with silicone hydrogel.

keratoconus in Malaysia. The results showed that RGP lenses significantly reduced aberration and provide better visual acuity than spectacles or SH contact lenses. The authors are aware that the small number of subjects may influence the results of this study. However, our findings are in agreement with earlier studies showing the superiority of RGP lenses over other types of refractive correction for enhancement of visual acuity in larger numbers of patients with keratoconus. Jinabhai et a ${ }^{30}$ compared the visual performance of 22 patients with keratoconus, and reported that RGP lenses provided superior visual performance than toric soft contact lenses. Griffiths et a $1^{15}$ compared the visual performance of RGP lenses against four different spherical contact lenses in 13 patients with keratoconus. Their results showed that RGP lenses provided better high-contrast and low-contrast visual acuity than the other soft contact lenses. In a report on three patients with keratoconus, Marsack et $\mathrm{al}^{31}$ showed that soft contact lenses (spherocylinder design) enabled less high-contrast and low-contrast visual acuity compared with RGP lenses. The improvement in visual acuity produced by RGP lenses is most likely attributable to the correction of irregular corneal astigmatism, ${ }^{32,33}$ and also to the greater reduction of optical aberrations induced by keratoconus. ${ }^{15}$ In comparison, larger amounts of residual corneal astigmatism could persist with soft contact lenses because these lenses mask and follow the distorted corneal contours. ${ }^{34}$

Our results show that contrast sensitivity scores with contact lens wear were higher than with spectacles. This finding confirms a previous report by Wei et $\mathrm{al}^{35}$ who studied contact lens characteristics and contrast sensitivity in patients with keratoconus. These authors reported that when compared with habitual correction, RGP lenses improved visual acuity significantly but patients still experienced a reduction in contrast sensitivity. Our results also show that SH and RGP lenses are able to correct ocular aberrations induced by keratoconus, with a better effect shown for RGP lenses. These findings corroborate those of previous studies comparing visual function and optical quality in patients with keratoconus using both soft and RGP lenses. ${ }^{13,15}$ Griffiths et $\mathrm{al}^{15}$ reported that RGP lenses significantly reduced corneal total aberrations (both lower-order and higher-order) when compared with four other types of spherical contact lenses evaluated in the same study. Moreover, Marsak et al ${ }^{13}$ reported that RGP lenses reduced total ocular RMS effectively when compared with spherocylinder soft contact lenses in patients with moderate to advanced keratoconus. Jinabhai et $\mathrm{al}^{24}$ also reported that RGP lenses reduced second-order cylinder RMS, third-order RMS, coma RMS, and trefoil RMS 
more effectively than soft toric contact lenses in their study of 22 patients with keratoconus. Similarly, in the present study, the RGP lens was found to significantly reduce the magnitude of total trefoil RMS and total coma RMS when compared with the SH lenses.

The present results also revealed no significant correlations between total ocular aberrations, higher-order aberrations, trefoil RMS, spherical RMS, or astigmatic RMS measured with $\mathrm{SH}$ lenses and the resulting visual acuity achieved. However, a negative correlation was found between coma RMS versus visual acuity and contrast sensitivity. These results support earlier findings by Jianbhai et $\mathrm{al}^{24}$ who reported a correlation between coma RMS and low-contrast acuity measured with toric soft contact lenses. Our findings are also in agreement with those of Fernandez-Sanchez et $\mathrm{al}^{36}$ who studied the effects of third-order RMS (coma and trefoil) induced by soft contact lenses on normal vision. These authors stated that large coma values (approximately $1 \mu \mathrm{m})$ significantly reduced visual performance. Statistical analysis of the present study also revealed moderate to strong negative correlations between higher-order aberrations, coma RMS, trefoil RMS, and astigmatic RMS measured with SH versus the contrast sensitivity scores achieved. However, such correlation was not observed using RGP lenses. It is clear that, of all the types of aberration, uncorrected coma RMS is associated with the poorest visual outcome in eyes with keratoconus, and the RGP lens minimized the magnitude of total and corneal aberrations, leading to an improvement in visual acuity and contrast sensitivity. ${ }^{5,37,38}$

According to the CLEK classification scale ${ }^{29}$ based on steep keratometric readings, the study subjects were categorized as having mild, moderate, and severe keratoconus. Unlike Jinabhai et al, ${ }^{24}$ who found a significant correlation between steep keratometric readings and both high-contrast and low-contrast acuity scores measured with toric soft lenses, the present study found no significant correlation between steep keratometric readings, average keratometric readings, and residual astigmatism measured with $\mathrm{SH}$ and RGP lenses and the resulting visual acuity achieved. Significant correlations were found only between steepest and average $\mathrm{K}$ readings measured without contact lenses and contrast sensitivity achieved with spectacles.

As most of the eyes in this study were categorized as having moderate to severe keratoconus, it is difficult to quantify the usefulness of SH contact lenses in the management of different grades of keratoconus. Overall, our results are in agreement with those reported by Jinabhai et al. ${ }^{30}$ In their comparative study of the optical quality of RGP lenses versus customized soft contact lenses in three patients with keratoconus, Jinabhai et al stated that soft material contact lenses appear likely to offer, at best, only minor visual improvement over RGP lenses for keratoconus.

The small number of subjects is the main limitation for this study. Larger numbers of patients with different degrees of severity of keratoconus are needed to confirm the findings of this investigation. However, our results concur with earlier investigations showing that contact lenses improve visual function and reduce aberrations in patients with keratoconus more than spectacles and therefore should be the first choice of management for patients with this condition.

\section{Acknowledgment}

This study was supported by research grants from Menicon Ltd Japan (NN022-2011) and Universiti Kebangsaan Malaysia (Industry-2011-030).

\section{Disclosure}

The authors report no conflicts of interest in this work.

\section{References}

1. Rabinowitz YS. Keratoconus. Surv Ophthalmol. 1998;42:297-319.

2. Marsack JD, Parker KE, Resudovs K, Donnelly WJ, Applegat RA. Uncorrected wavefront error and visual performance during RGP wear in keratoconus. Optom Vis Sci. 2007;84:463-470.

3. Davis LJ, Schechtman KB, Wilson BS, et al; CLEK study group. Longitudinal changes in visual acuity in keratoconus. Invest Opthalmol Vis Sci. 2006;47:489-500.

4. Maeda N, Fujikado T, Kuroda T, et al. Wavefront aberrations measured with Hartmann-Shack sensor in patients with keratoconus. Ophthalmology. 2002;109:1996-2003.

5. Xie PY, Wan D Yang LN, Zhou WJ. [The evaluation of visual quality in keratoconus eyes corrected by rigid gas-permeable contact lens]. Zhonghua Yan Ke Za Zhi. 2005;41:1086-1091. Chinese.

6. Lim L, Wei RH, Chan WK, Tan DT. Evaluation of higher order ocular aberrations in patients with keratoconus. $J$ Refract Surg. 2007;23: $825-828$.

7. Pantanelli S, MacRae S, Jeong TM, Yoon G. Characterizing the wave aberration in eyes with keratoconus or penetrating keratoplasty using a high-dynamic range wavefront sensor. Ophthalmology. 2007;114: 2013-2021.

8. Oshika T, Okamoto C, Samejima T, Tokunaga T, Miyata K. Contrast sensitivity function and ocular higher-order wavefront aberrations in normal human eyes. Ophthalmology. 2006;113:1807-1812.

9. Rocha KM, Vabre L, Harms F, Chateau N, Krueger RR. Effects of Zernike wavefront aberrations on visual acuity measured using electromagnetic adaptive optics technology. J Refract Surg. 2007;23: 953-959.

10. Okamoto C, Okamoto F, Samejima T, Miyata K, Oshika T. Higher order wavefront aberration and letter-contrast sensitivity in keratoconus. Eye (Lond). 2008;22:1488-1492.

11. Lawless M, Coster DJ, Phillips AJ, Loane M. Keratoconus: diagnosis and management. Aust N Z J Ophthalmol. 1989;17:33-60.

12. Barnett M, Mannis MJ. Contact lenses in the management of keratoconus. Cornea. 2011;30:1510-1516.

13. Negishi K, Kumanomido T, Utsumi Y, Tsubota K. Effect of higherorder aberrations on visual function in keratoconic eyes with a rigid gas permeable lens. Am J Ophthalmol. 2007;144:924-929. 
14. Jinabhai A, Radhakrishnan H, O’Donnell C. Visual acuity and ocular aberrations with different rigid gas permeable lens fitting in keratoconus. Eye Contact Lens. 2010;4:233-237.

15. Griffiths M, Zahner K, Collins MJ, Carney L. Masking of irregular corneal topography with contact lenses. CLAO J. 1998;24:76-81.

16. Rahi A, Davies P, Ruben M, Lobascher DJ, Menon J. Keratoconus and coexisting atopic disease. Br J Ophthalmol. 1977;61:761-764.

17. Betts AM, Mitchell GL, Zadnik K. Visual performance and comfort with the Rose K lens for keratoconus. Optom Vis Sci. 2002;79:493-501.

18. Edrington TB, Gundel RE, Libassi DP, et al; CLEK study group Variables affecting rigid contact lens comfort in the collaborative longitudinal evaluation of keratoconus (CLEK) study. Optom Vis Sci. 2004;81:182-188.

19. Lobascher DJ. Soft lenses in keratoconus. Contact Intraocul Lens Med J. 1975;1:23-27.

20. Koliopoulos J, Tragakis M. Visual correction of keratoconus with soft contact lenses. Ann Ophthalmol. 1981;13:835-837.

21. Gonzalez-Meijome JM, Jorge J, De Almeida JB, Parafita MA. Soft contact lenses for keratoconus: case report. Eye Contact Lens. 2006;32: 143-147.

22. Yamazaki ES, Da Silva VC, Morimitsu V, Sobrinho M, Fukushima N, Lipener C. [Keratoconus special soft contact lens fitting]. Arq Bras Oftalmol. 2006;69:557-560. Portuguese.

23. White L. Soft contact lenses for irregular cornea. Optom Pract. 2010;11 75-84.

24. Jinabhai A, Radhakrishnan H, Tromnas C, O’Donnell C. Visual performance and optical quality with soft lenses in keratoconus patients. Ophthalmic Physiol Opt. 2012;32:100-116.

25. Elliott DB, Sanderson K, Conkey A. The reliability of the Pelli-Robson contrast sensitivity chart. Ophthalmic Physiol Opt. 1990;10:21-24.

26. Agarwal A, Agarwal A, Jacob S. Textbook on Corneal Topography. New Delhi, India: Jaybee; 2010.

27. Hieda O, Kinoshita S. Measuring of ocular wavefront aberration in large pupils using OPD-scan. Semin Ophthalmol. 2006;18:35-40.
28. Bartsch DU, Bessho K, Gomez L, Freeman WR. Comparison of laser ray-tracing and skiascopic ocular wavefront-sensing devices. Eye (Lond). 2008;22:1384-1390.

29. Zadnik K, Barr JT, Gordon MO, Edrington TB. Biomicroscopic signs and disease severity in keratoconus. Cornea. 1996;15:139-146.

30. Jinabhai A, Charman WN, O'Donnell C, Radhakrishnan H. Optical quality for keratoconic eyes with conventional RGP lens and simulated, customised contact lens corrections: a comparison. Ophthalmic Physiol Opt. 2012;32:200-212.

31. Marsack JD, Parker KE, Applegate RA. Performance of wavefrontguided soft lenses in three keratoconus subjects. Optom Vis Sci. 2008;85: 1172-1178.

32. Jupiter DG, Katz HR. Management of irregular astigmatism with rigid gas permeable contact lenses. CLAO J. 2000;26:14-17.

33. Ozkurt Y, Atakan M, Gencaga T, Akkaya S. Contact lens visual rehabilitation in keratoconus and corneal keratoplasty. J Ophthalmol. 2012;2012:1-4.

34. Sorbara L, Chong T, Fonn D. Visual acuity, lens flexure, and residual astigmatism of keratoconic eyes as a function of back optic zone radius of rigid lenses. Cont Lens Anterior Eye. 2000;23:48-52.

35. Wei RH, Khor W, Lim L, Tan DT. Contact lens characteristics and contrast sensitivity of patients with keratoconus. Eye Contact Lens. 2011;37:307-311.

36. Fernandez-Sanchez V, Ponce ME, Lara F, Montes-Mico R, CastejonMochon JF, Lopez-Gil N. Effect of 3rd-order aberrations on human vision. J Cataract Refract Surg. 2008;34:1339-1344.

37. Egorov GB, Bobrovskikh NV, Savochkina OA. [Possibilities of compensation of optical aberrations in keratoconus with rigid gas-permeable contact lenses]. Vestn Oftalmol. 2010;126:42-46. Russian.

38. Shi YH, Wang LY, Lu TB, Qin J. [Changes of ocular higher order aberration in keratoconus eyes wearing rigid gas-permeable contact lens]. Zhonghua Yan Ke Za Zhi. 2011;47:601-606. Chinese.

\section{Clinical Optometry}

\section{Publish your work in this journal}

Clinical Optometry is an international, peer-reviewed, open access journal publishing original research, basic science, clinical and epidemiological studies, reviews and evaluations on clinical optometry. All aspects of patient care are addressed within the journal as well as the practice of optometry including economic and business analyses. Basic and clinical

\section{Dovepress}

research papers are published that cover all aspects of optics, refraction and its application to the theory and practice of optometry. The manuscript management system is completely online and includes a very quick and fair peer-review system, which is all easy to use. Visit http://www.dovepress. com/testimonials.php to read real quotes from published authors. 